\title{
Performance Evaluation of Routing Protocols for Voice and Video Traffics
}

\author{
S. Vasundra ${ }^{1}$ and D. Venkatesh ${ }^{2}$ \\ ${ }^{1 \& 2}$ Professor, Department of Computer Science and Engineering, \\ ${ }^{1}$ JNTUA College of Engineering, Anathapuramu, Andhra Pradesh, India \\ ${ }^{2}$ Anantha Lakshmi Institute of Technology and Sciences, Ananthapuramu, Andhra Pradesh, India \\ E-Mail: vasundras.cse@jntua.ac.in,dvvenkatesh@yahoo.co.in
}

\begin{abstract}
Wireless networks are comprised of nodes which are high in mobility and are adhoc natured where centralized access point is not required. Each node will have particular transmission range in which the data transmits from source to destination in that transmission range. Route construction for transmission of data from source to destination is tough due to high node mobility and dynamic nature of network. Many routing protocols are proposed and implemented in networks to reduce such mobility and dynamic difficulties for route construction. These routing protocols construct a route from source to destination based on availability of network nodes. Adhoc On demand Distance vector (AODV) is a self-starting dynamic network, Dynamic source routing (DSR) and Temporally Ordered Routing Algorithm (TORA) are most frequent used ones for dynamic route construction. Voice and Video are two mostly used applications nowadays as the users are immensely using them. In this paper, a comparison is made in performances of AODV, DSR and TORA for the traffics of voice and video. The performance is evaluated in terms of delay, throughput and packet delivery ratio. Simulations are conducted using network simulator NS 2.35.
\end{abstract}

Keywords: AODV, DSR, TORA, Routing Protocols, Voice and Video

\section{INTRODUCTION}

Wireless networking is the most common word for mobile users now a days. Wireless networks are two types. One which does have a need for infrastructure for communication and the other which does not need, the Ad hoc networks. Infrastructure networks are based on centralized station or wired access point.

Adhoc networks consists autonomous nodes which are selforganized. The reason for which the Adhoc networks are most used is that they have higher ubiquitous connectivity than that of internet. The wireless networks are easy to use and are flexible in nature. Different routing protocols are proposed for infrastructure networks believing that the network topology is static but these types of protocols do not serve with efficiency in case of wireless adhoc networks [1].

For adhoc networks two types of routing protocols are developed. They are table driven and on demand routing protocols. Table driven routing protocol maintains up-todate routing data from one node to any or all other nodes in the network. Each node thus maintains one or more routing table that stores the routes to all other network nodes. The problems of Table-driven routing protocol are hyperbolic signaling traffic and more importantly more power consumption because the routing data is disseminated to all other network nodes. [2]

On-demand routing protocol constructs and follows a special approach for transmission. A route is constructed when any of the nodes are ready to transmit the data. Once a source node $\mathrm{X}$ wants to transmit the date to node $\mathrm{Y}$, it invokes a routing discovery protocol to search out a route connecting it to $\mathrm{Y}$.

Once the route is found, nodes $\mathrm{X} \& \mathrm{Y}$ and every intermediate node store the route sequence from source node $\mathrm{X}$ to destination node $\mathrm{Y}$ and this is kept in the routing table. The constructed route is followed till the destination is unreached or the route is not any longer required. Ondemand routing protocols maintain lower power consumption. This protocol has long end-to-end delay because the connection is established only when the connection is required.

Now a day's more people prefer to use wi-fi networks since they are very readily available in low prices and easy to install. Different type of applications came to existence in parallel to the increasing of mobile users. Voice and video are most widely used applications among them. Around $84 \%$ of mobile users are using smart phones which can serve voice and video traffics for the needy.

The on-demand routing protocols AODV, DSR and TORA are most used in voice and video traffics for faster and reliable communication. AODV constructs the route from source to destination by sending a Route Request (RREQ) to all neighbors and stores this information in a table form. DSR constructs the route using route discovery and maintains the route through route maintenance process which is not available in AODV. TORA constructs a clear route by three processes which are route creation, route maintenance, and route erasure. This paper presents the literature survey in section II. Also explains about AODV, DSR and TORA in section III. Section IV consists of simulation setup and the results obtained while comparing 
the three protocols and finally Section $\mathrm{V}$ has a brief conclusion.

\section{LITERATURE SURVEY}

Several authors analyzed routing protocols and their performances in MANETs with different traffics. Naresh kumar [3] et al has presented the different aspects of routing protocols behavior. Pooja Sharma [4] et al. evaluated video streaming applications performance in vehicular adhoc networks. Rathy R.K[5] et al. compared AODV and DSR performances using group mobility model in random way point. AODV is superior to DSR in high quality and delay. Harminder S.B. [6] et al evaluated AODV and DSR routing protocol performance. This evaluation shows that the DSR performed well for TCP traffic when bandwidth is in limited condition. Elizabethm.

Royer [7] has stated different current routing protocols that are mostly used where TORA is considered one of the best routing protocol in on-demand routing protocols. P. Chenna Reddy [8] evaluated the sequential performances of AODV, DSR and TORA and concluded that in short lived nodes connectivity TORA performs better than the remaining two protocols in throughput. Rohini Sharma [9] evaluated the performances of the three protocols while searching for better energy optimization for adhoc networks and concluded that packet dropping rate that means the packet loss is very low in TORA compared to AODV and DSR which makes it suitable for better communication. Anuj K. Gupta.

[10] Evaluated AODV, DSR and TORA and concluded that when the nodes have higher mobility and shorter life time then TORA performs much better than AODV and DSR. The network throughput is comparatively better in TORA.

In this paper, the three routing protocols AODV, DSR and TORA performances are evaluated for the traffic sources voice and video. The performances evaluated are in terms of throughput, delay and packet delivery ratio. The work is aimed to find the behavior of AODV, DSR and TORA for when voice and video data are transmitted.

\section{DESCRIPTION OF ROUTING PROTOCOLS}

\section{A. $A O D V$}

In AODV, a route is made available only after requested by a network link and data about this route will be stored in all intermediate node's tables. The procedure of route establishment is as following. Let us assume that a node $\mathrm{X}$ needs to line up a connection with destination node $\mathrm{Y}$. The source node $\mathrm{X}$ initiates a path discovery method in an endeavor to determine a route to destination node Y. Here to find the route a Route Request (RREQ) packet is broadcasted by node $\mathrm{X}$ to its immediate neighbors. Each RREQ packet is known through a mix of the transmission node's informatics address and a broadcast ID. The latter is employed to spot totally different RREQ broadcasts by a similar node and is increased for every RREQ broadcast. Moreover, every RREQ packet has a sequence number that permits intermediate the nodes to reply to source requests solely.

Upon reception of associate RREQ packet by a node, the packet is sent to the immediate neighbors of the current node and this procedure continues till the RREQ finally gets to destination node $\mathrm{Y}$ or to a node which is recently linked a route to node Y. If any other copies of same RREQ packets are received by any node, they are discarded. Routing table records each and every node RREQ that has been forwarded to neighbors and the address of the neighbor node wherever the primary copy of the RREQ was received. This helps in constructing the reverse path, which is able to carry the response to the RREQ. AODV supports only the employment of symmetric links. A timer starts running once the route isn't used. If the timer moves out of the lifetime value, then the route entry is deleted [11].

\section{B. DSR}

Dynamic Source Routing (DSR) Dynamic Source Routing (DSR) uses source node to find the route from source to destination. [12] In the order list of network nodes DSR uses each packet in its header to be routed carries. This means that the packet which is having the route is to be relayed. Thus, intermediate nodes need not maintain routing information because the contents of the packet itself are enough to send a packet in a route. This truth eliminates the requirement for the periodic route management and neighbor detection packets. The overhead in DSR is massive as every packet must contain the complete sequence of nodes with route composition.

DSR comprises route discovery and route maintenance processes. A supply node wishes to set up a link to a different node initiates the route discovery method by broadcasting a RREQ packet. The neighboring nodes receive the packet and successively forward it to their own neighbors. A RREQ message is forwarded by a node given that it's not nevertheless been seen by this node if the nodes address isn't a part of route. A route reply packet (RREP) is initiated by RREQ packet when it receives the RREQ packet by destination node which have a clear route to the destination. If RREQ message arrived at the destination or to associate intermediate node then that particular node is aware of a route to the destination and the replied packet contains the nodes sequence that represent the final route. This info is piggybacked on to the RREP message and consequently created offered at the sender node [13].

DSR supports each even and asymmetric links. Thus, the RREP messages are often either carried over a similar path with original RREQ, or the destination node would possibly initiate its own route discovery method towards finding the source node. In this process, they send back the RREP message in its request that is sent. To limit the overhead of 
those management messages, every node will have a cache will store routes that were either utilized by these nodes or overheard. Thus, a RREQ process could lead to variety of routes being hold on within the sender node's cache. The sender node initiates Route maintenance upon detection of an amendment in network topology that fore stalls its packet from reaching the destination node. In such situations, the source node will either decide to use various routes to the destination node or reinitiate route discovery. Storing within the table of different routes means route discovery are often avoided when alternative routes are available. DSR can be quicker than the other on-demand routing protocols. DSR does not create the use the transmission period or routing information because the route maintenance is initiated before.

\section{TORA}

Temporally Ordered Routing Algorithm (TORA) TORA enables link only when required so it is a reactive routing algorithm. TORA is similar to that of Light Weight Mobile Routing (LMR). TORA basically follows link reversal as well as route repair procedures which are similar to LMR.TORA detects partitions which are no productive reversal links and stops them. The network topology in TORA is of Directed Graph that means the flow in it follows a direction. TORA mainly contains three operations which are a) Route Creation b) Route Maintenance and c) Route erasure. TORA has A Directional Acyclic Graph (DAG) Network which maintains its nodes in terms of heights. Each node in DAG network is assigned with height metric $h$. For example, the node $i$, is written as hi and node $j$ is defined as hj. The link direction for these is denoted as hi $>$ hj. The height in TORA is named as quintuple. This quintuple will be comprised of time, node ID, new reference level, reflection indicator, ordering parameters and next node ID.

Route Creation initially makes destination node height to 0 and all the other nodes it makes to NULL which in terms means undefined. As soon as the setting of heights is done the source node sends a query (QRY) packet to all the nodes. This QRY packet will be having the destination ID. The nodes which have no-NULL values replies by sending reverse update (UPD) packet which contains height of them. After sending UPD the node itself changes its height to one more than previous height they had. The nodes with maximum height is placed at top of DAG and lower heights at the lower end. By taking heights, the graph is constructed which defines a route from source to destination. TORA works when having high node mobility in the network.

Sometimes the link might get damaged due to its high mobility so a need for route maintenance is needed. TORA maintains a set of nodes which have control messages where the node topology might change at the regular intervals or in a sudden change. If a node suddenly loses the link then the node makes a new reference and sends the new reference to all neighbor nodes. They revert the link topology change and maintain the continuation of new reference link. TORA has additional operation which is route erase. In this operation TORA sends clear (CLR) packets for the entire network and deletes the existing invalid routes.

\section{SIMULATION SETUP AND RESULTS}

Network simulator NS2.35 is used to conduct different simulations that are needed to compare the three routing protocols. In this simulation 50 random positioned nodes are taken. These random nodes will be continuously moving in high frequency within the range. From these 50 nodes 10 nodes are taken as source purpose and 10 nodes are taken as destination purpose. All the 10 source nodes are generating voice and video traffics. Voice traffic is generated by VoIP application and Video data is generated by VBR. The data rate for voice is $256 \mathrm{kbps}$ and for video is $512 \mathrm{kbps}$ to $1 \mathrm{mbps}$.

The packet size taken is 1000B. All nodes are starting their transmission at 0 th sec. The time taken for simulation is 200 sec. This simulation aims at denoting the traffics of voice and video on the three routing protocols AODV, DSR and TORA and compares their performances. 1. Performance metrics since more number of parameters available to evaluate the performance, for the simulation purpose only three parameters which are important for voice and video traffics are considered. They are throughput, Packet delivery ratio and end to end delay.

\section{A. Throughput}

Throughput in simple terms is stated as number of bits transmitted per sec.

\section{B. Packet Delivery Ratio}

In particular time period, the number of packets received by the destination node to that of the number of packets send by source node is called as packet delivery ratio.

\section{End-to-End Delay}

It is calculated on the average time taken for packet to reach from source node to destination node. Using the simulation which is setup routing protocols are taken and considerably the performances of them are compared. The three ondemanding protocols which are AODV, DSR and TORA performances are noted down. Each and every performance metric which is discussed in simulation setup is calculated for voice and video traffics.

In case of voice traffic source in Fig 1, at 0m/s AODV, DSR, TORA gives equal and maximum throughput. At later stages of simulations AODV and DSR gives less throughput than TORA.

With video traffic source in Fig. 2, at $0 \mathrm{~m} / \mathrm{s}$ DSR gives more throughput than AODV and TORA but as simulation time 
increases throughput of DSR decreases. In the same way the throughput of AODV decreases than that of TORA. Thus, TORA is better than that of both AODV and DSR.

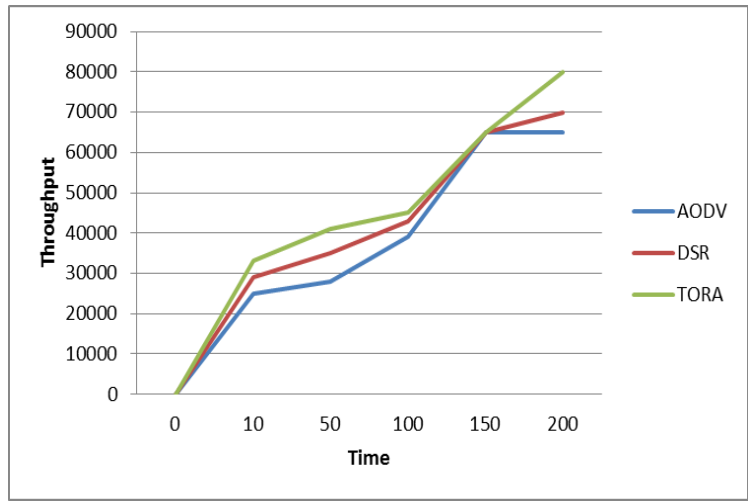

Fig. 1 Simulation time vs Throughput for voice

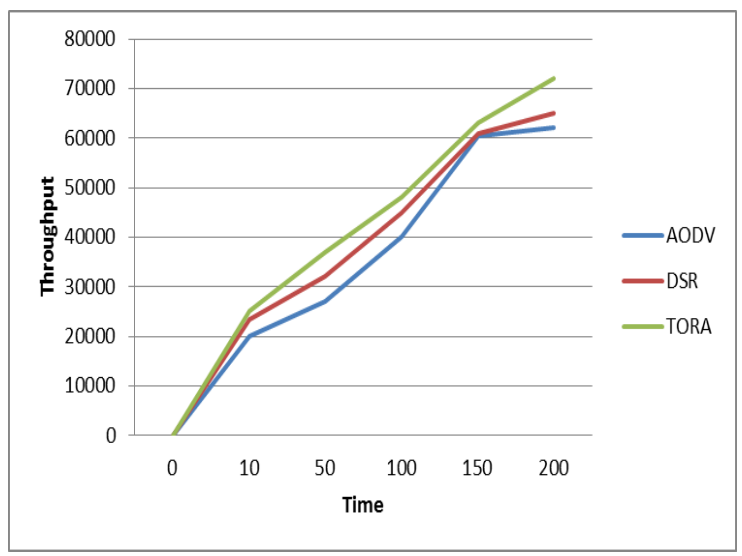

Fig. 2 Simulation time vs Throughput for video

With video traffic source in Fig. 2, at $0 \mathrm{~m} / \mathrm{s}$ DSR gives more throughput than AODV and TORA but as simulation time increases throughput of DSR decreases. In the same way the throughput of AODV decreases than that of TORA. Thus, TORA is better than that of both AODV and DSR.

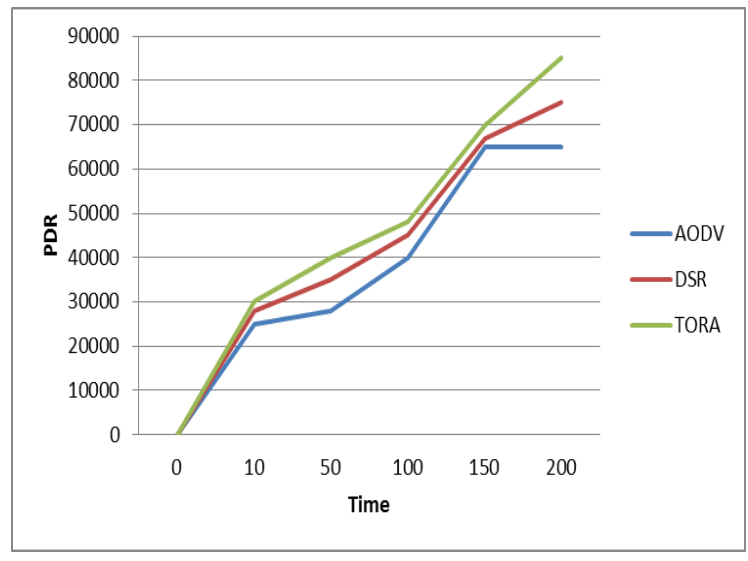

Fig. 3 Time vs PDR for voice traffic

In case of voice traffic source at earlier stages of simulation DSR and AODV performs better than TORA. But as the time increases DSR and AODV performs equally under full load condition but less than TORA as shown in fig 3 .

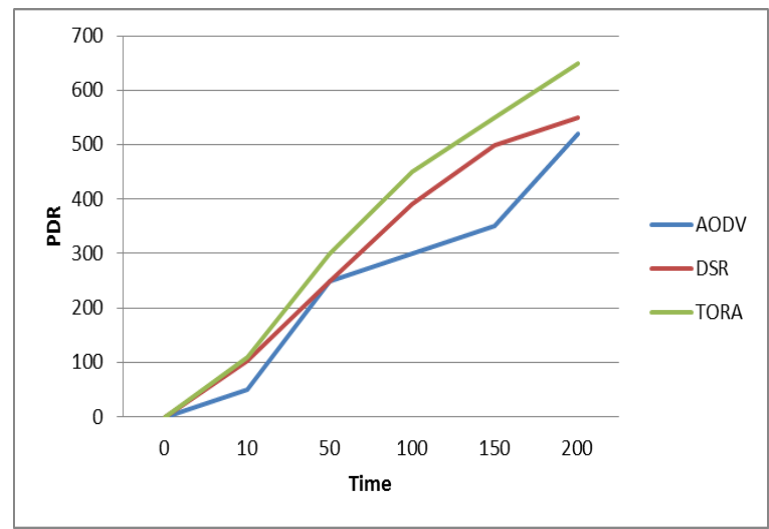

Fig. 4 Time vs PDR for Video traffic

This shows that even in this metric also TORA outperforms both. With video, DSR and AODV gives more PDR than TORA at both low as well as late stages of simulations. At earlier stages TORA is comparable to AODV and DSR but the ratio decreases as the time increases as shown in fig 4 .

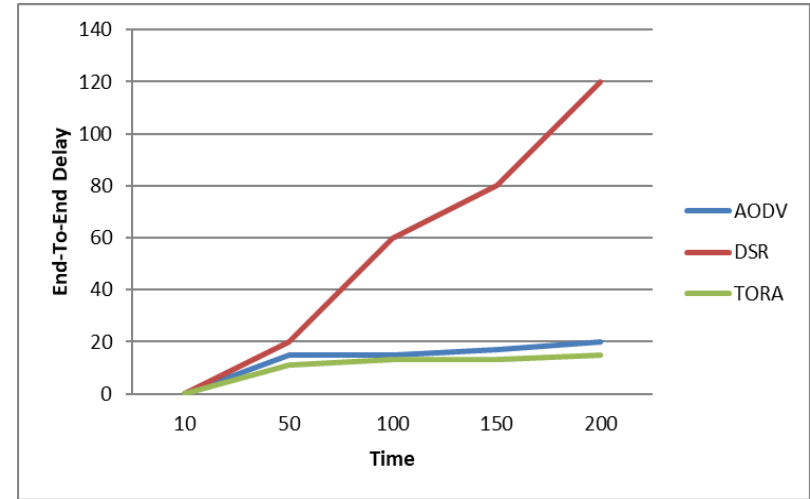

Fig. 5 Delay vs time for voice

In case of voice, TORA is mostly constant and least delay at all the time.

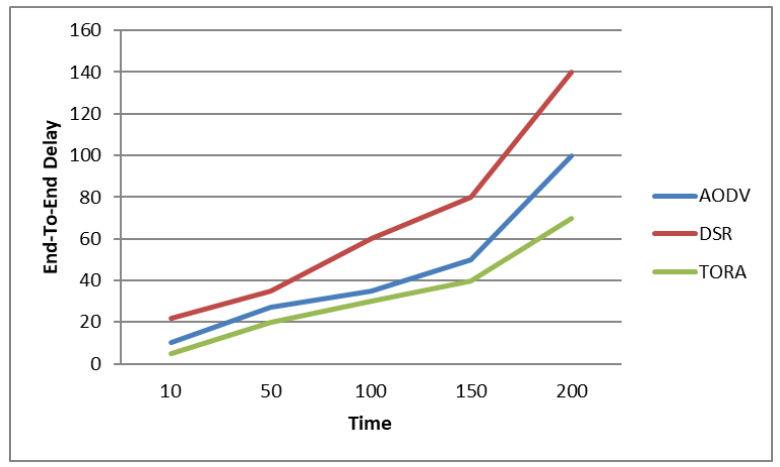

Fig. 6 Delay vs time for video traffic

When delay of AODV and DSR is taken they considerably increases with time. 
In case of video also, TORA gives less delay than AODV and DSR. Thus, the average end-to-end delay is least for TORA routing protocol with voice and video than DSR.

\section{CONCLUSION}

Simulations for the three routing protocols AODV, DSR and TORA are conducted with voice and video traffic sources. Supported the results it is tended to conclude that, each AODV, DSR and TORA offers nearly same packet delivery ratio at earlier stages of simulations DSR gives higher PDR with video. Delay is most for DSR followed by AODV and minimum for TORA with video. Average endto-end delay of TORA is a smaller than AODV and DSR. In fact, TORA is better than AODV and DSR. Thus, TORA with video traffic outperforms AODV and DSR. In this paper the routing protocols AODV, DSR and TORA's are analyzed and their performances are calculated for few performance metrics for two traffic sources since voice and video are widely used applications in mobile technology.

\section{REFERENCES}

[1] G. N. Vivekananda, and P. Chenna Reddy, "Performance evaluation of TCP, UDP, and SCTP in MANETS," ARPN Journal of Engineering and Applied Sciences, Vol.13, No.9, pp. 3087-3092, 2018.

[2] Yu, Yan, Ramesh Govindan, and Deborah Estrin, "Geographical and energy aware routing: A recursive data dissemination protocol for wireless sensor networks," Stanford Semantic Scholar, 2001.

[3] S. Umamaheswaran, Naresh Kumar, and P. K. Biswas, "A survey of routing protocol design patterns for mobile ad hoc networks," IEEE 2nd International Conference on Emerging Technology Trends in Electronics, Communication and Networking (ET2ECN),2014.

[4] Sharma, Pooja, Ajay Kaul, and M. L. Garg, "Performance analysis of video streaming applications over VANETs," International Journal of Computer Applications, Vol. 112, No.14, 2015.

[5] Kumar, Suresh, R. K. Rathy, and D. Pandey, "Design of an ad-hoc network model for disaster recovery scenario using various routing protocols," Proceedings of the International Conference on Advances in Computing, Communication and Control, ACM, 2009.

[6] S. Bindra, Harminder , Sunil K. Maakar, and A. L. Sangal, "Performance evaluation of two reactive routing protocols of MANET using group mobility model," International Journal of Computer Science, Vol.7, No.3, pp.38-43, 2010.

[7] Royer, M. Elizabeth, and Chai-Keong Toh, "A review of current routing protocols for ad hoc mobile wireless networks," IEEE Personal Communications, Vol.6, No.2, pp.46-55, 1999.

[8] P. C. Reddy, P. C. Reddy, "Performance analysis of adhoc network routing protocols," Ad Hoc and Ubiquitous Computing, pp. 186-187, 2006.

[9] Sharma, Rohini, and D.K.Lobiyal, "Energy holes avoiding techniques in sensor networks: A survey," International Journal of Engineering Trends and Technology, Vol. 20, No. 4, pp.204-208, 2015.

[10] Gupta, K.Anuj, Harsh Sadawarti, and Anil K.Verma, "Performance analysis of AODV, DSR \& TORA routing protocols," International Journal of Engineering and Technology, Vol. 2, No. 2, 2010.

[11] I.Broustis, G.Jakllari, T.Repantis, and M.Molle, "A Comprehensive Comparison of Routing Protocols for Large-Scale Wireless MANETs," In SECON conference, Vol. 6, pp. 951-956, September, 2006.

[12] Lee, Sung-Ju, Mario Gerla, and Chai-Keong Toh, "A simulation study of table-driven and on-demand routing protocols for mobile ad hoc networks," IEEE network, Vol. 13, No. 4, pp. 48-54, 1999.

[13] Moravejosharieh, and Amirhossein, "Performance Analysis of AODV, AOMDV, DSR, DSDV Routing Protocols in Vehicular Ad Hoc Network," Research Journal of Recent Sciences, 2013. 